\title{
Numerical bifurcation diagram for the two-dimensional boundary-fed chlorine-dioxide-iodine-malonic-acid system
}

\author{
S. Setayeshgar* and M. C. Cross \\ Condensed Matter Physics 114-36, California Institute of Technology, Pasadena, California 91125
}

(Received 22 June 1998)

\begin{abstract}
We present a numerical solution of the chlorine-dioxide-iodine-malonic-acid reaction-diffusion system in two dimensions in a boundary-fed system using a realistic model. The bifurcation diagram for the transition from nonsymmetry-breaking structures along boundary feed gradients to transverse symmetry-breaking patterns in a single layer is numerically determined. We find this transition to be discontinuous. We make a connection with earlier results and discuss prospects for future work. [S1063-651X(99)01104-6]
\end{abstract}

PACS number(s): 82.20.Wt, 82.20.Mj, 47.54.+r

\section{INTRODUCTION}

In 1952, Turing showed [1] that under certain conditions, reaction and diffusion processes alone could lead to the symmetry-breaking instability of a system from a homogeneous state to a stationary patterned state. The Turing instability is characterized by an intrinsic wavelength, in contrast to hydrodynamic instabilities, such as Rayleigh-Bénard convection where the wavelength depends on cell height. For this reason, this instability mechanism has particular relevance to pattern formation in biological systems [2,3]. Given the difficulties of noninvasive experiments on biological systems, experimental studies of Turing pattern formation have been carried out primarily on chemical systems. Even so, Turing patterns have been generated in the laboratory only recently, specifically in the chlorite-iodidemalonic-acid (CIMA) chemical reaction-diffusion system [4-7].

Although Turing patterns have been extensively studied theoretically and numerically in the context of abstract models, the possibility of comparison with controlled and reproducible experiments provides motivation for quantitative analyses based on realistic models of these systems. Lengyel, Rabai, and Epstein (henceforth referred to as LRE) have proposed a realistic model of the simpler chlorine dioxideiodine-malonic acid (CDIMA) reaction-diffusion system $[8,9]$. The chemistry of the CDIMA and CIMA systems are related, and the two are similar in terms of their stationary pattern forming and dynamical behavior. The potential for both experimental and theoretical work makes the CDIMA system well suited for the study of nonequilibrium pattern formation in general.

In practice, this has not been fully realized, and unlike in fluid systems [10], experimental and theoretical efforts in chemical systems have not been closely coupled. First, numerical solution of reaction-diffusion equations using realistic chemical parameters is computationally demanding. In addition, the algebraic complexity of the realistic nonlinear

\footnotetext{
*Author to whom correspondence should be addressed. Present address: Applied Mathematics Department, Caltech, CA. FAX: 626 683 3549. Electronic address: simas@ama.caltech.edu
}

reaction terms renders these models unsuitable for analysis by standard analytical tools. Furthermore, unlike the case considered originally by Turing and subsequently by others, the experimental system is not uniform. A continuous supply of reactants is fed into the reactor from two separately nonreactive reservoirs to keep the system far from equilibrium, setting up gradients in the concentrations of these reservoir species along the width of the reactor. In an earlier work [11], we used the realistic LRE model to investigate the formation of one-dimensional stationary structures along the boundary feed gradients and their linear instability to transverse symmetry-breaking (Turing) patterns. Here, we extend these results by numerically solving the fully nonlinear reaction-diffusion equations in two dimensions.

Kadar, Lengyel, and Epstein [12] have also numerically investigated two-dimensional Turing patterns within the LRE model, but in a closed system (i.e., in the absence of gradients) where the patterns are transient by nature. Twodimensional numerical simulations of Turing patterns in ramped systems have been performed using popular abstract models, such as the Schnakenberg model $[13,14]$, and the Brusselator model [15-17], as well as the generalized SwiftHohenberg equation [18]. These models, which are easier to implement, produce patterns that possess similar features to those observed in experimental systems. However, they do not allow quantitative comparison or prediction of new features at specific parameter values of a real chemical system.

In this article, we present a numerical solution of the LRE model in a boundary-fed system in two dimensions, corresponding to sustained patterns in thin-strip open reactors. We determine the branch of the bifurcation diagram corresponding to the transition to stripes in this system, a result that can be directly investigated in experiments based on the CDIMA system. In Sec. II, we present the model and parameters used. In Sec. III, we describe our numerical method. We present our results in Sec. IV, and give conclusions and prospects for future work in Sec. V.

\section{CHEMICAL MODEL}

The LRE model of the CDIMA reaction-diffusion system has been discussed in detail elsewhere $[12,11,19]$. The resulting governing partial differential equations are given below: 


$$
\begin{aligned}
\frac{\partial[\mathrm{MA}]}{\partial t} & =-r_{1}+D_{\mathrm{MA}} \nabla^{2}[\mathrm{MA}], \\
\frac{\partial\left[\mathrm{I}_{2}\right]}{\partial t} & =-r_{1}+\frac{1}{2} r_{2}+2 r_{3}-r_{4}+D_{\mathrm{I}_{2}} \nabla^{2}\left[\mathrm{I}_{2}\right], \\
\frac{\partial\left[\mathrm{ClO}_{2}\right]}{\partial t} & =-r_{2}+D_{\mathrm{ClO}_{2}} \nabla^{2}\left[\mathrm{ClO}_{2}\right], \\
\frac{\partial\left[\mathrm{I}^{-}\right]}{\partial t} & =r_{1}-r_{2}-4 r_{3}-r_{4}+D_{\mathrm{I}^{-}} \nabla^{2}\left[\mathrm{I}^{-}\right], \\
\frac{\partial\left[\mathrm{ClO}_{2}^{-}\right]}{\partial t} & =r_{2}-r_{3}+D_{\mathrm{ClO}_{2}^{-}} \nabla^{2}\left[\mathrm{ClO}_{2}^{-}\right], \\
\frac{\partial\left[\mathrm{SI}_{3}^{-}\right]}{\partial t} & =r_{4} .
\end{aligned}
$$

The nonlinear reaction terms are given by

$$
\begin{aligned}
& r_{1}=\frac{k_{1 a}[\mathrm{MA}]\left[\mathrm{I}_{2}\right]}{k_{1 b}+\left[\mathrm{I}_{2}\right]}, \\
& r_{2}=k_{2}\left[\mathrm{ClO}_{2}\right]\left[\mathrm{I}^{-}\right], \\
& r_{3}=k_{3 a}\left[\mathrm{ClO}_{2}^{-}\right]\left[\mathrm{I}^{-}\right]\left[\mathrm{H}^{+}\right]+\frac{k_{3 b}\left[\mathrm{ClO}_{2}^{-}\right]\left[\mathrm{I}_{2}\right]\left[\mathrm{I}^{-}\right]}{h+\left[\mathrm{I}^{-}\right]^{2}}, \\
& r_{4}=k_{+}[\mathrm{S}]\left[\mathrm{I}_{2}\right]\left[\mathrm{I}^{-}\right]-k_{-}\left[\mathrm{SI}_{3}^{-}\right] .
\end{aligned}
$$

The left/right reservoir species are malonic acid (MA)/chlorine dioxide $\left(\mathrm{ClO}_{2}\right)$ and iodine $\left(\mathrm{I}_{2}\right)$, respectively. As these species diffuse through and react in the gel reactor, the dynamical iodide $\left(\mathrm{I}^{-}\right)$and chlorite $\left(\mathrm{ClO}_{2}^{-}\right)$species are produced. The concentration of starch in excess $(S)$ is assumed to be uniform and constant, equal to its initial value $\left(S_{0}\right)$. The starch triiodide complex $\left(\mathrm{SI}_{3}{ }^{-}\right)$is the experimentally observed species.

The rate and diffusion constants used in the numerical calculations here are taken from Refs. $[12,19,20]$ and are given in Table I. The value of the parameter $h$ in Eq. (9) is not an experimentally determined quantity but rather chosen in an ad hoc manner $[8,21]$ to preserve the validity of the second term in the rate law $r_{3}$ for very low $\left[\mathrm{I}^{-}\right]$. This issue is discussed in detail by Rudovics et al. [22]. In the twosided-feed geometry explored here, at the boundaries where Dirichlet boundary conditions hold for the iodide species, this rate law will not be strictly valid. However, our earlier work [11] demonstrates its validity $\left(\left[\mathrm{I}^{-}\right] \geq \sqrt{h}\right)$ in the relevant interior of the gel (and away from the boundaries) where the localized front patterns form.

The experimental CIMA reaction is similar to the CDIMA reaction in terms of dynamics and stationary pattern formation $[19,23,24]$. However, a quantitative model of the CIMA reaction does not exist. In particular, the role of chlorinedioxide in this reaction is not well understood [19]. Furthermore, it has been pointed out that in the experimental CIMA system, use of chlorite and iodide along with acid as reservoir species could lead to reactive reservoirs [19]. In the
TABLE I. Kinetic constants for the CDIMA system.

\begin{tabular}{ccc}
\hline \hline Rate or diffusion constant & Dimensions & Value \\
\hline$k_{1 a}$ & $\left(\mathrm{~s}^{-1}\right)$ & $9 \times 10^{-4 \mathrm{a}}$ \\
$k_{1 b}$ & $(\mathrm{M})$ & $5 \times 10^{-5} \mathrm{a}$ \\
$k_{2}$ & $\left(\mathrm{M}^{-1} \mathrm{~s}^{-1}\right)$ & $1 \times 10^{3} \mathrm{a}$ \\
$k_{3 a}$ & $\left(\mathrm{M}^{-2} \mathrm{~s}^{-1}\right)$ & $1.2 \times 10^{2} \mathrm{a}$ \\
$k_{3 b}$ & $\left(\mathrm{~s}^{-1}\right)$ & $1.5 \times 10^{-4} \mathrm{a}$ \\
$h$ & $\left(\mathrm{M}^{2}\right)$ & $1.0 \times 10^{-14} \mathrm{a}$ \\
$k_{+}$ & $\left(\mathrm{M}^{-2} \mathrm{~s}^{-1}\right)$ & $6.0 \times 10^{5} \mathrm{~b}$ \\
$k_{-}$ & $\left(\mathrm{s}^{-1}\right)$ & $1.0^{\mathrm{b}}$ \\
$D_{\mathrm{I}^{-}}$ & $\left(\mathrm{cm}^{2} \mathrm{~s}^{-1}\right)$ & $7.0 \times 10^{-6} \mathrm{c}$ \\
$D_{\mathrm{ClO}_{2}^{-}}$ & $\left(\mathrm{cm}^{2} \mathrm{~s}^{-1}\right)$ & $7.0 \times 10^{-6} \mathrm{c}$ \\
$D_{\mathrm{I}_{2}}$ & $\left(\mathrm{~cm}^{2} \mathrm{~s}^{-1}\right)$ & $6.0 \times 10^{-6} \mathrm{a}$ \\
$D_{\mathrm{MA}}$ & $\left(\mathrm{cm}^{2} \mathrm{~s}^{-1}\right)$ & $4.0 \times 10^{-6} \mathrm{a}$ \\
$D_{\mathrm{ClO}_{2}}$ & $\left(\mathrm{~cm}^{2} \mathrm{~s}^{-1}\right)$ & $7.5 \times 10^{-6} \mathrm{a}$ \\
$D_{\mathrm{H}^{+}}$ & $\left(\mathrm{cm}^{2} \mathrm{~s}^{-1}\right)$ & $1.0 \times 10^{-5}$ \\
$K[\mathrm{~S}]_{\mathrm{o}}$ & $\left(\mathrm{M}^{-1}\right)$ & $6.25 \times 10^{4} \mathrm{~d}$ \\
\hline \hline
\end{tabular}

${ }^{\mathrm{a}}$ From [20] at $7^{\circ} \mathrm{C}$.

${ }^{b}$ From [12] at $4^{\circ} \mathrm{C}$.

${ }^{\mathrm{c}}$ From [23] at $4^{\circ} \mathrm{C}$.

${ }^{\mathrm{d}}$ From [19] at $4{ }^{\circ} \mathrm{C}$.

CDIMA system, chlorine dioxide is nonreactive with both iodine and malonic acid, allowing for well controlled boundary concentrations of these species. It is known from batch experiments and simulations of the CDIMA system that concentrations of chlorine dioxide, iodine, and malonic acid vary little on the scale of variations in the chlorite and iodide concentrations [8]. This observation has formed the basis of the adiabatic elimination of the former reactants, resulting in a two-variable reduction of the full model $[8,25]$, and making their identification as the background species a natural one. Hence, in the interest of aligning experiment with numerical and theoretical work in this area, it appears reasonable for experiments to implement the CDIMA system.

\section{NUMERICAL METHOD}

A pseudospectral method was used to solve the governing partial differential equation in two dimensions. The physical boundary conditions are no flux in the $x$ direction (transverse to the gradients), and fixed point boundary conditions in the $z$ direction (along the gradients). In our numerical implementation, the governing equations are cosine Fourier transformed in the $x$ direction, and each spectral mode is evolved in time as a one-dimensional problem in the $z$ direction. We used a five-point finite-difference approximation with a variable-width spatial mesh to allow better resolution of the sharp front patterns along the gradients. The time-stepping scheme was Crank-Nicolson for the linear terms (implicit) and Adams-Bashford for the nonlinear terms (explicit), both second-order accurate. After each time step, the updated solutions were transformed back into real space to reconstruct the nonlinear terms.

As Table I indicates, the large order-of-magnitude variations in the real chemical parameters of the LRE model make numerical solution of this model less tractable than the afore- 
(a)

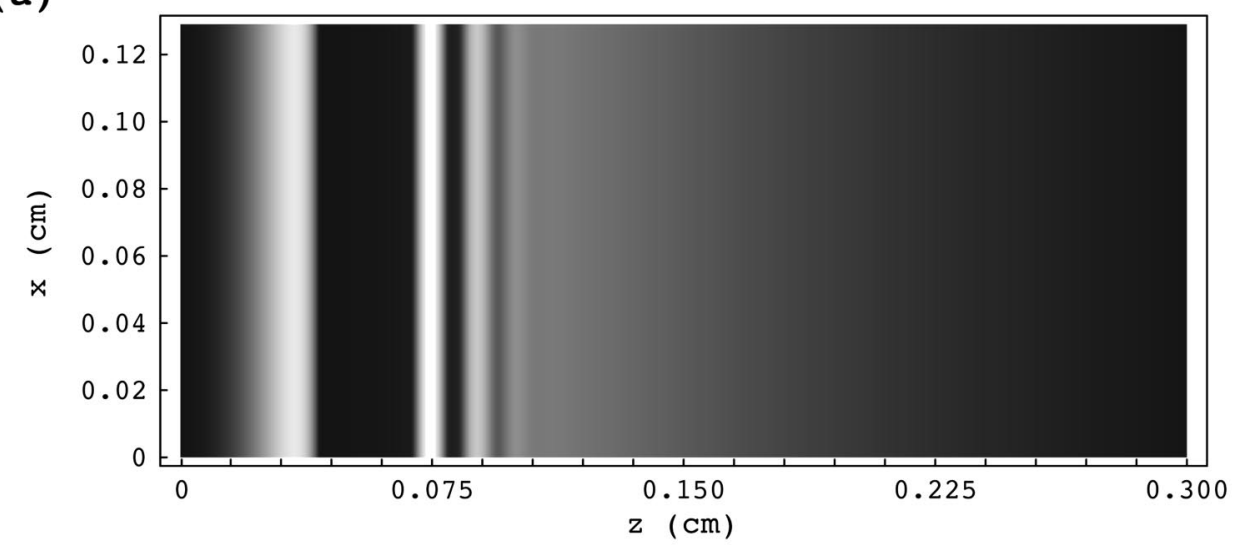

(b)

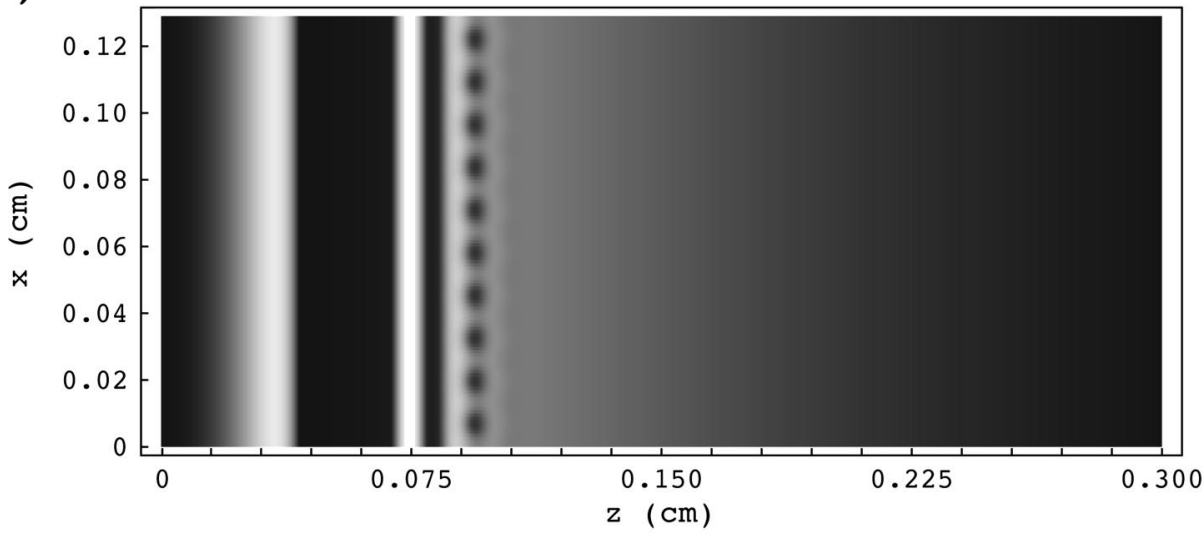

FIG. 1. Two-dimensional solution for starch triiodide with $[\mathrm{MA}]_{L}=0.0115 \mathrm{M}$ : The initial condition is shown in (a). The numerical solution after $T=1000 \mathrm{~s}$ of evolution time is shown in (b). Dark and light shadings correspond to low and high concentrations, respectively.

mentioned abstract models. The stability restriction on the time step $\Delta t$ due to explict treatment of the nonlinear terms is onerous. Hence, we parallelized the above numerical scheme, and the computations were performed on a 512node Intel Paragon.

As initial conditions, we used the stationary solution $\vec{u}_{s}(z)$ along $z$ with a uniform profile in $x$, to which we added a perturbation given by the most linearly unstable mode $k^{*}$ :

$$
\vec{u}(x, z, t=0)=\vec{u}_{s}(z)+C \overrightarrow{\delta u}_{k} *(z) \cos \left(k^{*} x\right) .
$$

$\overrightarrow{\delta u}_{k^{*}}(z)$ is the eigenvector obtained from the linear stability analysis, and $C$ is an overall scale factor to ensure that the perturbation is small and lies in the linear regime. The concentration vectors correspond to the six variables of the LRE model. The full six-variable linear stability analysis was carried out using inverse iteration [26]. The nonzero boundary conditions in the $z$ direction are $[\mathrm{MA}]_{\mathrm{L}}=0.0115 \mathrm{M},\left[\mathrm{ClO}_{2}\right]_{\mathrm{L}}=0.006 \mathrm{M}$, and $\left[\mathrm{I}_{2}\right]_{\mathrm{L}}$ $=0.008 \mathrm{M}$, where $(R, L)$ refer the right and left reservoirs, respectively. From the linear stability analysis of $\vec{u}_{s}(z)$, the growth rate for the instability at $k^{*}=471.2 \mathrm{~cm}^{-1}$ is $\lambda\left(k^{*}\right)$ $=0.00465 \mathrm{~s}^{-1}$, giving a characteristic saturation time of $\tau$ $\sim 1 / \lambda \sim 215 \mathrm{~s}$. The system size is $0.3 \mathrm{~cm}$ in the $z$ direction and $0.133 \mathrm{~cm}$ in the $x$ direction, corresponding to exactly ten wavelengths in the $x$ direction. The spatial resolution of the system investigated here was $N_{x}=129$ and $N_{z}=914$. One thousand iterations at this resolution required approximately 12 node hours.
The integration time step was $\Delta t=0.001 \mathrm{~s}$, chosen to be the same as that for the time evolution in one dimension. In the one-dimensional time evolution, the restriction on the time step due to the explicit treatment of the nonlinear terms was explored empirically, by varying $\Delta t$ and using a value such that the algorithm was stable. We investigated using a higher-order (third-order) Adams-Bashford scheme to verify that the restriction on $\Delta t$ was limited by stability as opposed to accuracy. The dynamically evolved stationary state was compared with that obtained from direct solution using a Newton-Raphson root-finding algorithm. We determined the time step used in one dimension to be adequate for the time evolution in two dimensions as follows: By using initial conditions uniform in the $x$ direction (and equal to $5 \times 10^{-12} \mathrm{M}$ for all species), thereby reducing the two-dimensional time evolution to be effectively one-dimensional, we verified that the asymptotic solution was the same as that obtained in one dimension. It is possible that implementing a numerical algorithm adapted to solving stiff partial differential equations (see Ref. [12] and references therein) will improve the total execution time in the two-dimensional evolution.

\section{RESULTS}

\section{A. Two-dimensional stationary solution}

In Fig. 1, we show density plots of the initial state, as well as the numerical solution for the starch triiodide species after a total integration time of approximately $T=1000 \mathrm{~s}$, with 

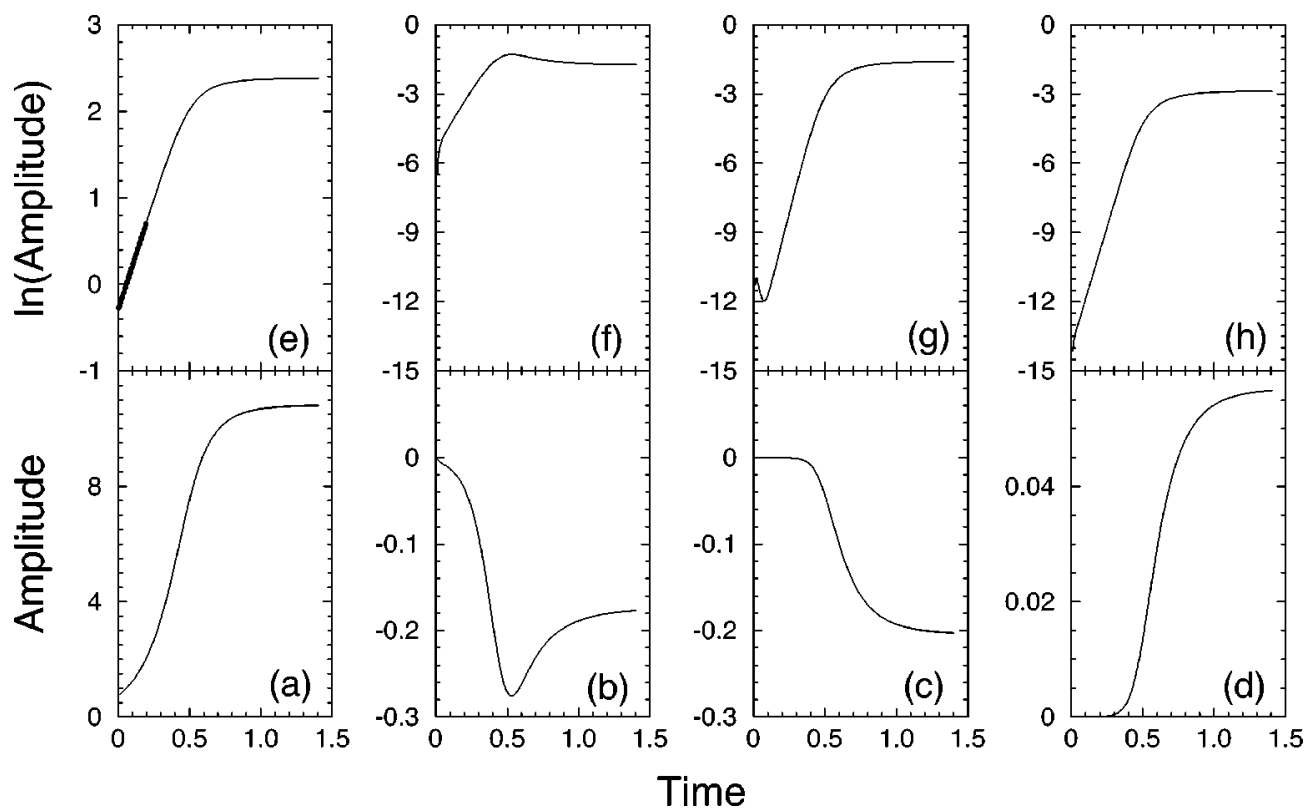

FIG. 2. Time evolution of the most linearly unstable mode $k^{*}$ and its higher-order harmonics: (a)-(d) show the values of the $k^{*}, 2 k^{*}, 3 k^{*}$, and $4 k^{*}$ modes for the starch triiodide species at the peak of the most linearly unstable eigenvector $(z=0.094 \mathrm{~cm})$, as a function of time; (e)-(h) show the logarithms of magnitudes of these amplitudes. The plots are shown for nondimensionalized quantities. (The time and concentration conversion factors are $9 \times 10^{-4} \mathrm{~s}^{-1}$ and $5 \times 10^{-5} \mathrm{M}$, respectively.) The slope of the linear segment (heavy line) in plot (e) for $t<0.2$ is $5.129 \pm 0.012$, and agrees well with the growth rate $\lambda\left(k^{*}\right)=5.172$ from the linear stability analysis.

dark and light shading representing low and high concentrations, respectively. In Figs. 2(a)-(d), we show the time evolution of the $k^{*}$ mode and its higher-order harmonics at the peak of the linear instability eigenvector $(z=0.094 \mathrm{~cm})$ for the starch triiodide species. In Figs. 2(e)-(h), we plot the logarithm of the magnitude of these quantities. (These plots are shown for the nondimensionalized quantities.) The slope of the linear segment in Fig. 2(e), for $t<\tau \sim 0.2$ is 5.129 \pm 0.012 (corresponding to $0.004616 \pm 0.000011 \mathrm{~s}$ ) agrees well (to within one percent) with the linear growth rate, and further verifies our linear stability results. In these plots, it is apparent that the saturated amplitudes of the higher-order harmonics are much smaller than that of $k^{*}$.

To compare the size of the nonlinear perturbations in the $x$ direction with the unperturbed profile in the $z$ direction, we have plotted the $x$ and $z$ dependence of the two-dimensional solution. In Fig. 3(a), we show the $x$ dependence at the peak of the linear instability eigenvector $(z=0.094 \mathrm{~cm})$, which approximates a pure Fourier mode, verified by the relatively small saturated amplitudes of the higher-order harmonics in Fig. 2. In Fig. 3(b), the dashed and dotted lines denote the profiles in the $z$ direction at $x=0.067 \mathrm{~cm}=5 \lambda$ and $x$ $=0.073 \mathrm{~cm}=5.5 \lambda$, respectively. The solid line denotes the profile of the unperturbed one-dimensional stationary state. We note that although the saturated amplitude of the transverse instability is comparable to the variation of the onedimensional stationary state in the $z$ direction, its $x$ dependence is not strongly nonlinear.

The results presented in this section can be summarized by three points. First, we have presented a numerical solution of two-dimensional patterns in the boundary-fed CDIMA reaction-diffusion system using the LRE model. Second, our numerical solution agrees qualitatively with patterns observed in thin-strip reactors in experiments on the CIMA system, for experimental conditions giving a single unstable front [27]. The wavelength of the solution presented here is $0.133 \mathrm{~mm}$, in agreement with the $0.13-0.33 \mathrm{~mm}$ experimentally observed range [28]. Finally, the numerical evolution in two dimensions confirms our result from the linear stability analysis of the one-dimensional stationary state along the gradients [11].

\section{B. Bifurcation diagram}

The symmetry-breaking transition from Fig. 1(a) to Fig. 1 (b) is effectively one dimensional, since only a single layer is unstable over a range of values of $[\mathrm{MA}]_{\mathrm{L}}$ control parameter, as we showed in Ref. [11]. In this earlier work, we demonstrated the existence of (three) disjoint unstable ranges as the $[\mathrm{MA}]_{\mathrm{L}}$ control parameter was continuously varied from $4.0 \times 10^{-3} \mathrm{M}$ to $4.0 \times 10^{-2} \mathrm{M}$, consistent with experimental observations in thin-strip reactors showing the appearance and subsequent vanishing of a transverse instability as one of reservoir concentrations was increased. Here, we numerically investigate the dependence of the saturated amplitude of the transverse instability on the $[\mathrm{MA}]_{\mathrm{L}}$ control parameter in the vicinity of the (lower) bifurcation point for one of these unstable ranges $\left(9.73 \times 10^{-3} \mathrm{M}<[\mathrm{MA}]_{\mathrm{L}}\right.$ $\left.<1.25 \times 10^{-2} \mathrm{M}\right)$. In the following, for convenience, we report our results in nondimensionalized units: the time conversion factor is $k_{1 a}=9 \times 10^{-4} \mathrm{~s}^{-1}$, and the concentration conversion factor is $k_{1 b}=5 \times 10^{-5} \mathrm{M}$.

First, the critical control parameter was determined numerically from a linear fit to the maximum linear growth rate versus $[\mathrm{MA}]_{\mathrm{L}}$. This value was found to be $[\mathrm{MA}]_{\mathrm{c}}$ $=194.5226$. Linear stability analysis of the stationary state at this value of $[\mathrm{MA}]_{\mathrm{L}}$ yields a maximum growth rate of $\lambda^{*}$ $=1.1054 \times 10^{-4}$. This value of $\lambda^{*}$, which is expected to be zero, gives a combined measure of the numerical uncertainties in the determination of the stationary state at a particular 

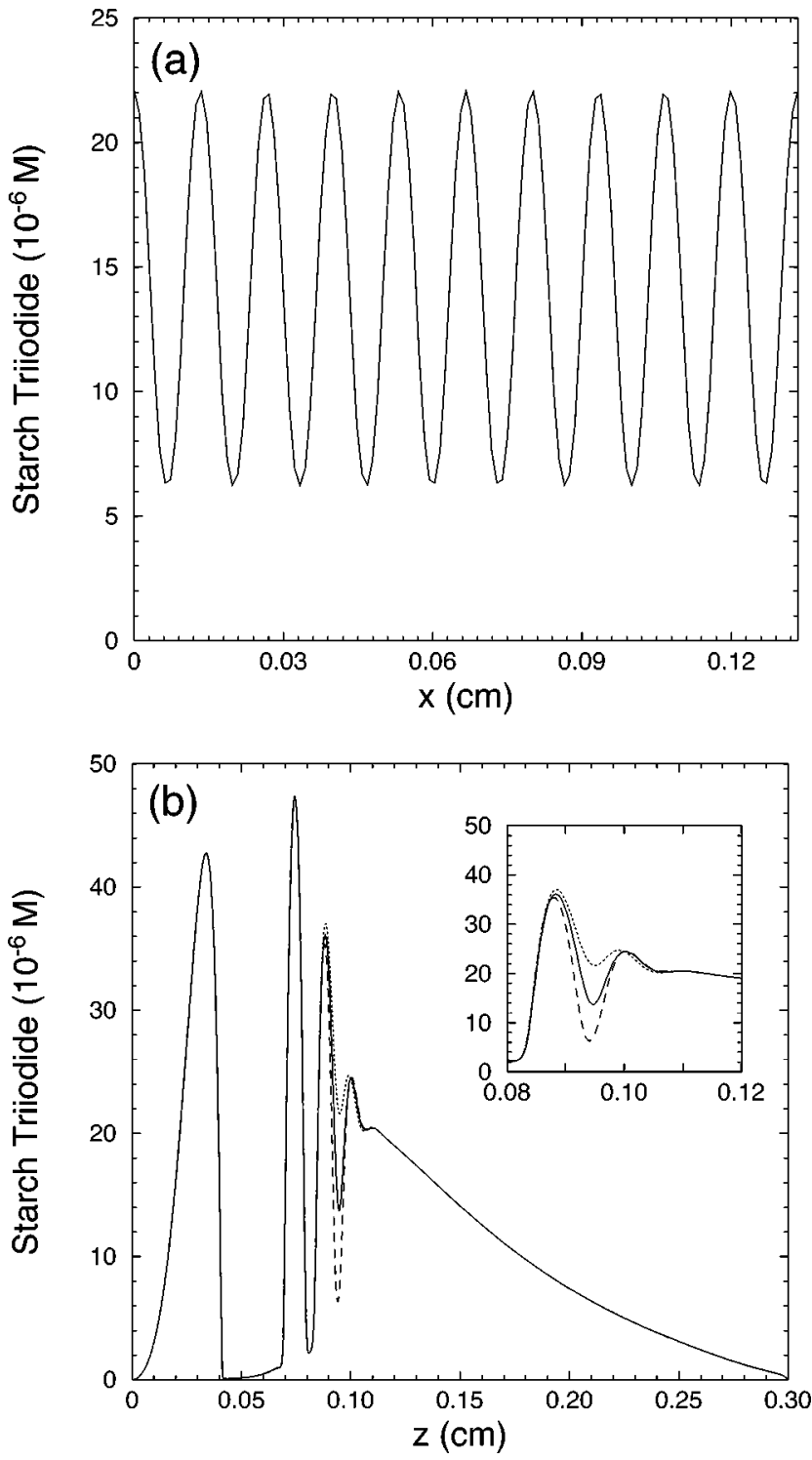

FIG. 3. Spatial dependence of the two-dimensional solution in the $x$ and $z$ directions for $\mathrm{SI}_{3}^{-}$species: (a) The system size in the $x$ direction is $0.133 \mathrm{~mm}$, constructed to incorporate exactly ten wavelengths of the most linearly unstable mode $k^{*}$. The profile in the $x$ direction is plotted at $z=0.094 \mathrm{~cm}$. (b) The dashed and dotted lines denote the profiles in the $z$ direction at $x=0.067 \mathrm{~cm}=5 \lambda$ and $x$ $=0.073 \mathrm{~cm}=5.5 \lambda$, respectively. The solid line denotes the profile of the unperturbed one-dimensional stationary state. We note that the saturated amplitude of the instability is comparable to the variation in the profile of the one-dimensional stationary state in the $z$ direction.

value of $[\mathrm{MA}]_{\mathrm{L}}$ as well its linear stability. Hence, in principle, there would be error bars associated with values of $[\mathrm{MA}]_{\mathrm{L}}$, equal to $\Delta[\mathrm{MA}]_{\mathrm{L}}=a \Delta \lambda=3.0826 \times 10^{-4}$, where $a$ is the slope of the linear fit from which $[\mathrm{MA}]_{\mathrm{c}}$ is extracted.

Figure 4 shows the computed bifurcation diagram. Starting in the supercritical regime, for each value of $[\mathrm{MA}]_{\mathrm{L}}$, as initial condition, we use the corresponding one-dimensional stationary solution in the $z$ direction seeded with the most linearly unstable eigenvector at approximately the saturated amplitude of the previous higher value of control parameter (adiabatic approach). The final converged amplitudes were obtained from a least squares fit of the dynamical evolution

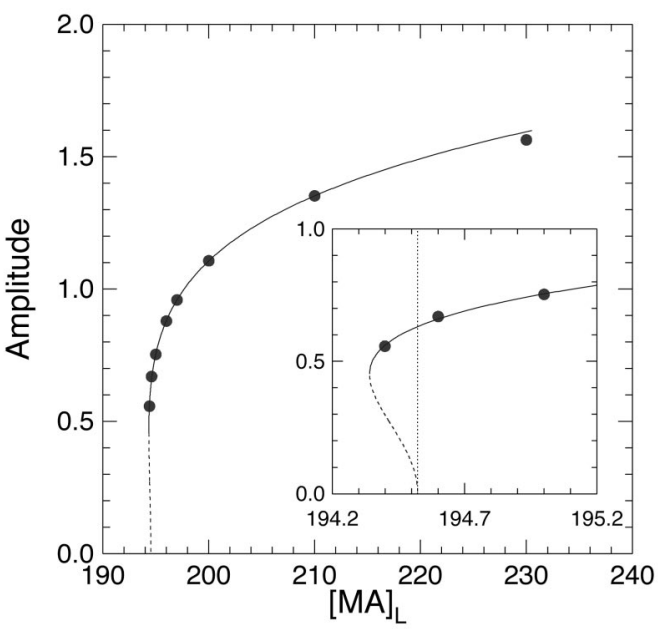

FIG. 4. Bifurcation diagram: The solid curve is the computed fit; the broken curve corresponds to the unstable branch. The inset shows the vicinity of the saddle node bifurcation ( $[\mathrm{MA}]_{\mathrm{SN}}$ $=194.34)$, and the dotted vertical line denotes linear threshold $\left([\mathrm{MA}]_{\mathrm{c}}=194.5229\right)$. The plots are shown for nondimensionalized quantities.

of the peak amplitude to an exponential plus a constant offset, excluding initial points until the converged value did not depend on the number of excluded points.

Close to onset, the solution is given by

$$
\vec{u}(x, z, t)=\overrightarrow{\delta u}_{k^{*}}(z)\left[A(t) \exp \left(i k^{*} x\right)+A^{*}(t) \exp \left(-i k^{*} x\right)\right],
$$

where $k^{*}$ is the most linearly unstable mode, and $A(t), A^{*}(t)$ are complex conjugates. The amplitude equation, which gives a universal description of the weakly nonlinear behavior and depends only on the symmetries of the problem (in this case, translational invariance in the $x$ direction) is (at seventh order):

$$
\frac{\partial A}{\partial t}=\epsilon A+g_{1}|A|^{2} A+g_{2}|A|^{4} A+g_{3}|A|^{6} A,
$$

where the coefficients depend on the specific system under investigation, and $\epsilon \equiv[\mathrm{MA}]_{\mathrm{L}}-[\mathrm{MA}]_{\mathrm{c}}$ is the distance from onset. Our results, described below, reveal a subcritical (first order) bifurcation.

Figure 4 shows a sixth order polynomial fit to the numerical data: $[\mathrm{MA}]_{\mathrm{L}}=[\mathrm{MA}]_{\mathrm{c}}-g_{1}|A|^{2}-g_{2}|A|^{4}-g_{3}|A|^{6}$. First, $[\mathrm{MA}]_{\mathrm{c}}$ was held fixed at the linear threshold value, and $\left(g_{1}, g_{2}, g_{3}\right)$ were fitted for. The goodness of the fit depended on the number of points farthest from linear threshold retained in the fit. We determined that excluding the last point $\left([\mathrm{MA}]_{\mathrm{L}}=230.0\right)$ gave the best fit. The fitted parameters are

$$
\begin{aligned}
& g_{1}=1.7585, \\
& g_{2}=-4.0825, \\
& g_{3}=-0.82677 .
\end{aligned}
$$

Since $g_{1}>0$, the instability does not saturate for $\epsilon>0$, and the bifurcation is subcritical. We also investigated allowing 


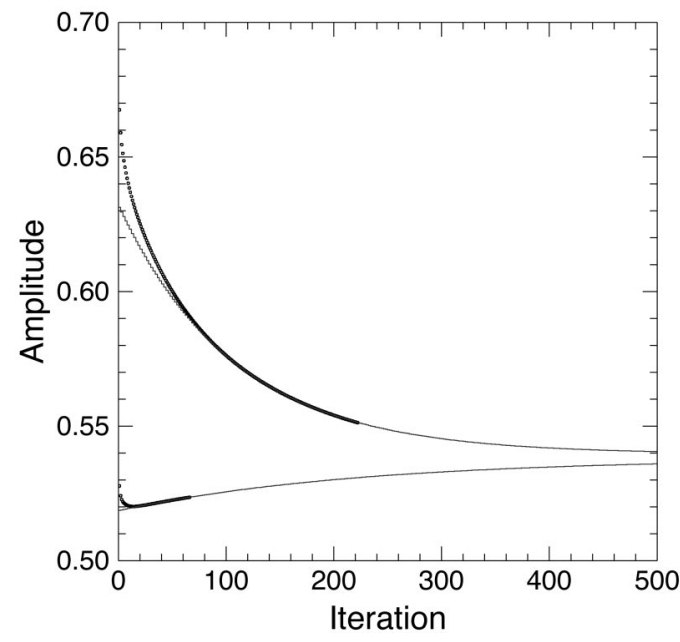

FIG. 5. Convergence to finite amplitude below linear threshold, $[\mathrm{MA}]_{\mathrm{L}}=194.4$ : The closely spaced circles denote the numerical time evolution, and the solid lines denote the computed fit to an exponential plus a constant offset. Convergence from above and below to a finite amplitude is apparent. The plot is shown for nondimensionalized amplitude.

all parameters, $\left([\mathrm{MA}]_{c}, g_{1}, g_{2}, g_{3}\right)$, to float. Again, excluding the last point produces a fit with an offset $[\mathrm{MA}]_{\mathrm{c}}$, which is closest to the linear threshold value. The values of the fitted parameters in this case are $\left([\mathrm{MA}]_{\mathrm{c}}, g_{1}, g_{2}, g_{3}\right)$ $=(194.47,1.5548,-3.8692,-0.89160)$.

Figure 5 shows the convergence of the peak amplitude of the fastest growing linear mode for $[\mathrm{MA}]_{\mathrm{L}}=194.4$ in the subcritical region. It shows convergence from above and below to a finite amplitude instability. The error for the converged amplitude is estimated to be one-half of the difference between the converged-from-above and convergedfrom-below values. This error $\left(8.0 \times 10^{-4}\right)$ is taken to be the same for all points, even though the convergence from below was not repeated for all points [29]. We also confirmed the decay of a linear perturbation at this same subcritical value. An exponential fit to the dynamical evolution of the peak amplitude of the instability yields a decay rate of $\lambda=-0.0416$, in good agreement with the largest eigenvalue, $\lambda=-0.0429$.

The minimum value of $[\mathrm{MA}]_{\mathrm{L}}$ below which a finite amplitude instability does not exist, corresponding to the saddle-node bifurcation, can be computed from the fitted parameters. Using the parameter values given in Eqs. (14)(16), $[\mathrm{MA}]_{\mathrm{SN}}$ is found to be

$$
[\mathrm{MA}]_{\mathrm{SN}}=194.34 .
$$

The inset in Fig. 4 shows this turning point. For $[\mathrm{MA}]_{\mathrm{L}}$ $=193.0$ below this value, we explicitly verified decay to zero of an initial perturbation with amplitude $A=0.7756$.

We note that the transition is "weakly" subcritical. This is characterized by the small range of control parameter below linear threshold, approximately equal to 0.18 (9 $\times 10^{-6} \mathrm{M}$ ), for which a finite amplitude instability exists, in comparison with the linearly unstable range, 55.4 (2.77 $\left.\times 10^{-3} \mathrm{M}\right)$, determined in our earlier work [11]. The weakly subcritical nature of the transition implies that a linear stability analysis of the one-dimensional structures along the gradients [11] does have utility in predicting the existence of a transverse instability for given reaction parameters and boundary conditions over a wide range of control parameters (in the supercritical regime).

\section{CONCLUSION AND DISCUSSION}

To summarize, we have carried out a two-dimensional numerical simulation of the boundary-fed CDIMA reactiondiffusion system based on the realistic LRE model for this system. Our results are qualitatively similar to those seen in experiments on the CIMA reaction-diffusion system, and support the earlier work [11] in which we studied the linear instability of the boundary-fed CDIMA system to transverse Turing patterns.

Numerical studies by Jensen et al. [30-32] on the twovariable LRE model with uniform backgrounds have found the transition to stripes in one and two dimensions to be subcritical. Our results demonstrate this transition to also be subcritical in the boundary-fed LRE model. This prediction and computed bifurcation diagram can be directly verified by experiments based on the CDIMA system.

The subcritical nature of the transition to stripes makes the LRE model qualitatively different from other abstract reaction-diffusion models hitherto used to study Turing patterns. For example, in the ramped Brusselator, the transition to stripes has been shown to be supercritical [33]. Jensen et al. have investigated the propagation of fronts separating the homogeneous steady state from the Turing structure in one and two dimensions using the uniform LRE model. The subcriticality allows for the existence of a range of values of control parameters for which the front velocity vanishes, allowing an infinite number of stable steady inhomogeneous structures. Despite the weakly subcritical nature of the transition, it would be interesting to similarly investigate front propagation and formation of localized (quasi-onedimensional) states in the boundary-fed system.

In experimental geometries (disk reactors) where the dimensions of the reactor transverse to the gradients are large, the analog of the one-dimensional row of spots that develops in our numerical simulation and in experiments using thinstrip reactors is a two-dimensional "monolayer." Dufiet et al. [34] have pointed out that these monolayers, which are confined by a strong transverse gradient of reservoir chemical concentrations, must be distinguished from genuine twodimensional structures with uniform control parameters. Pattern selection in genuine two- and three-dimensional systems has been studied analytically and numerically using abstract reaction-diffusion models [35]. However, it is not practical to generate sustained genuine structures experimentally. In the context of a model reaction-diffusion system, Dufiet et al. have shown that in genuine two-dimensional systems and monolayers, the stripe-hexagon competition is similar close to onset. They find, however, that far from onset, hexagonal phases in monolayers are restabilized due to their interaction with a longitudinal $(k=0)$ instability. The latter finding is consistent with earlier theoretical predictions $[36,37]$, as well as experiments in bevelled disk reactors [38]. 
It would be interesting to numerically investigate pattern selection for monolayers in the LRE model of the CDIMA system in the range of boundary conditions and reaction parameters accessible to experiments, allowing in principle direct comparison with experimental results. This would require extension of our numerical computation to three dimensions.

\section{ACKNOWLEDGMENTS}

This work was supported by the National Science Foundation under Grant No. DMR 9311444, and by a generous award of computer time from the Center for Advanced Computing Research at Caltech. We thank Ruben Krasnopolsky for invaluable advice with the parallelization.
[1] A. M. Turing, Philos. Trans. R. Soc. London, Ser. B 327, 37 (1952).

[2] H. Meinhardt, Models of Biological Pattern Formation (Academic Press, London, 1982).

[3] J. D. Murray, Mathematical Biology (Springer-Verlag, Berlin, 1989), Chap. 15.

[4] V. Castets, E. Dulos, J. Boissonade, and P. De Kepper, Phys. Rev. Lett. 64, 2953 (1990).

[5] P. De Kepper, V. Castests, E. Dulos, and J. Boissonade, Physica D 49, 161 (1991).

[6] Q. Ouyang and H. L. Swinney, Nature (London) 352, 610 (1991).

[7] Q. Ouyang and H. L. Swinney, in Chemical Waves and Patterns, edited by R. Kapral and K. Showalter (Kluwer, Dordrecht, 1995), p. 269.

[8] I. Lengyel, G. Rabai, and I. R. Epstein, J. Am. Chem. Soc. 112, 4606 (1990).

[9] I. Lengyel, G. Rabai, and I. R. Epstein, J. Am. Chem. Soc. 112, 9104 (1990).

[10] M. C. Cross and P. C. Hohenberg, Rev. Mod. Phys. 65, 851 (1993).

[11] S. Setayeshgar and M. C. Cross, Phys. Rev. E 58, 4485 (1998).

[12] S. Kadar, I. Lengyel, and I. R. Epstein, J. Phys. Chem. 99, 4504 (1995).

[13] V. Dufiet and J. Boissonade, Physica A 188, 158 (1992).

[14] V. Dufiet and J. Boissonade, J. Chem. Phys. 96, 664 (1992).

[15] P. Borckmans, A. De Wit, and G. Dewel, Physica A 188, 137 (1992).

[16] G. Dewel, P. Borckmans, A. De Wit, B. Rudovics, J. J. Perraud, E. Dulos, J. Boissonade, and P. De Kepper, Physica A 213, 181 (1995)

[17] P. Borckmans, G. Dewel, A. De Wit, and D. Walgraef, in Chemical Waves and Patterns (Ref. [7]).

[18] M. F. Hilali, S. Metens, P. Borckmans, and G. Dewel, Phys. Rev. E 51, 2046 (1995).

[19] I. Lengyel and I. R. Epstein, in Chemical Waves and Patterns (Ref. [7]), p. 297.

[20] I. Lengyel and I. R. Epstein, Science 251, 650 (1991).
[21] D. M. Kern and D. H. Kim, J. Am. Chem. Soc. 87, 5309 (1992).

[22] R. Rudovics, E. Barillot, P. Davies, E. Dulos, B. Boissonade, and P. De Kepper (unpublished). These authors report experimental results in a new, one-sided-feed geometry that respects the validity of the rate law given by Eq. (9) everywhere, and consequently could be better suited than the two-sided-feed geometries hitherto explored for quantitative studies of the CDIMA system.

[23] I. Lengyel, S. Kardar, and I. P. Epstein, Phys. Rev. Lett. 69, 2729 (1992).

[24] I. Lengyel, S. Kadar, and I. R. Epstein, Science 259, 493 (1993).

[25] I. Lengyel and I. R. Epstein, Proc. Natl. Acad. Sci. USA 89, 3977 (1992).

[26] S. Setayeshgar, Ph.D. thesis, California Institute of Technology, 1998.

[27] J. Boissonade, E. Dulos, and P. De Kepper, in Chemical Waves and Patterns (Ref. [7]), p. 221.

[28] Q. Ouyang and H. L. Swinney, Chaos 1 (4), 411 (1991).

[29] This convergence from above and below was also confirmed for a point in the supercritical region, $[\mathrm{MA}]_{\mathrm{L}}=195.0$, giving even better agreement between the two fitted converged values, with an error of $\left(1.0 \times 10^{-4}\right)$.

[30] O. Jensen, V. O. Pannbacker, G. Dewel, and P. Borckmans, Phys. Lett. A 179, 91 (1993).

[31] O. Jensen, V. O. Pannbacker, E. Mosekilde, G. Dewel, and P. Borckmans, Phys. Rev. E 50, 736 (1993).

[32] O. Jensen, E. Mosekilde, P. Borckmans, and G. Dewel, Phys. Scr. 53, 243 (1996).

[33] J. Boissonade, J. Phys. (France) 49, 541 (1988).

[34] V. Dufiet and J. Boissonade, Phys. Rev. E 53, 4883 (1996).

[35] A. De Wit, G. Dewel, P. Borckmans, and D. Walgraef, Physica D 61, 289 (1992).

[36] C. B. Price, Phys. Lett. A 194, 385 (1994).

[37] G. Dewel, S. Metens, M. F. Hilali, P. Borckmans, and C. B. Price, Phys. Rev. Lett. 74, 4647 (1995).

[38] E. Dulos, P. Davies, B. Rudovics, and P. De Kepper, Physica D 98, 53 (1996). 